\title{
Optical orientation and spin-dependent recombination in GaAsN alloys under continuous-wave pumping
}

\author{
E L Ivchenko ${ }^{1}$, V K Kalevich ${ }^{1}$, A Yu Shiryaev ${ }^{1}$, \\ M M Afanasiev ${ }^{1}$ and Y Masumoto ${ }^{2}$ \\ 1 A.F. Ioffe Physico-Technical Institute, St. Petersburg 194021, Russia \\ 2 Institute of Physics, University of Tsukuba, Tsukuba 305-8571, Japan \\ E-mail: kalevich@solid.ioffe.ru
}

\begin{abstract}
We present a systematic theoretical study of spin-dependent recombination and its effect on optical orientation of photoelectron spins in semiconductors with deep paramagnetic centers. For this aim we generalize the Shockley-Read theory of recombination of electrons and holes through the deep centers with allowance for optically-induced spin polarization of free and bound electrons. Starting from consideration of defects with three charge states we turn to the two-charge-state model possessing nine parameters and show that it is compatible with available experimental data on undoped GaAsN alloys. In the weak- and strong-pumping limits, we derive simple analytic equations which are useful in prediction and interpretation of experimental results. Experimental and theoretical dependencies of the spin-dependent-recombination ratio and degree of photoluminescence circular polarization on the pumping intensity and the transverse magnetic field are compared and discussed.
\end{abstract}

PACS numbers: 72.20.Jv, 72.20.Bh, 72.25.Fe, 78.55.-m

Submitted to: J. Phys: Condens. Matter 


\section{Introduction}

In recent years proposals for quantum computation and spintronics have initiated search for semiconductor nonmagnetic bulk materials and heterostructures that might be used to realize high values of electron spin polarization, on the one hand, and its long storage time, on the other. At low temperatures, this task is successfully realized by exploiting low spin-relaxation rates of charge carriers localized in semiconductor quantum wells or confined in self-organized quantum dots [1, 2, 3, 4,. With increasing temperature up to room temperature the free carriers get delocalized and, in zinc-blende-lattice bulk semiconductors, their spin relaxation time drastically decreases to values of the order of 100 ps or even less [3, 5, 6, 7] which limits the electron spin polarization under continuouswave (cw) photoexcitation to few percent [3, 5. The situation can be partially improved in strongly $n$-doped samples where the intense electron-electron collisions essentially suppress the D'yakonov-Perel' mechanism of spin relaxation, as shown theoretically [8] and experimentally [9], see also a comprehensive review article by $\mathrm{Wu}$ et al [10]

Recent experimental studies of electron spin dynamics in GaAsN alloys have revealed strong contradiction with the existing expectations: extremely high spin polarization, up to $90 \%$, of the free electrons and its preservation during $\sim 1 \mathrm{~ns}$ have been found at room temperature in nonmagnetic undoped GaAsN bulk films and GaInAsN quantum wells [11, 12, 13, 14, 15, 16, 17, 18, 19]. In these experiments electron spin polarization was created by optical pumping and detected by polarized photoluminescence (PL). It has been established that the anomalously high polarization and spin memory arise due to spin-dependent recombination of free electrons with deep paramagnetic centers present in the nitrogen alloys and subsequent dynamic spin polarization of the centers [13, 14, 16, 18]. The latter acts as a spin filter preventing the spin majority photoelectrons to recombine and promoting the spin minority photoelectrons to disappear from the conduction band. As a result the total density of photoelectrons increases superlinearly with the increasing pumping power which leads to an enhancement of the edge PL intensity (up to a factor of 8) [13, 20, 21] as well as of room-temperature photoconductivity [22, 23]. Recent measurements of optically detected magnetic resonance (ODMR) [21, 24] and optical orientation of electron spins [14, 18, have provided a proof that the paramagnetic centers in question are $\mathrm{Ga}_{\mathrm{i}}$ self-interstitial defects and established the critical dependence of the defect concentration on the $\mathrm{N}$ composition, $\mathrm{Ga}(\mathrm{In}) \mathrm{AsN}$ alloy growth conditions and postgrowth treatment, e.g., thermal annealing.

The spin-dependent recombination (SDR) gives rise to formation of a coupled spin system of free and bound electrons where the free-electron polarization is intricately related to the spin state of paramagnetic centers. For the quantitative description of this coupled nonlinear system we proposed the SDR model [13, 16] based on the model introduced by Weisbuch and Lampel [25] and used later by Paget [26] in a qualitative analysis of low-temperature optical orientation of electron spins in GaAlAs and GaAs, respectively. Our model contains nine parameters. At present most of them 
have been found for GaAsN experimentally. Particularly, the measurements of cw and time-resolved polarized luminescence under circularly-polarized photoexcitation in the presence of a transverse magnetic field have allowed to estimate the spin-relaxation times of free and bound electrons, lifetime of photoholes, absolute value of free-electron $g$ factor and signs of the free- and bound-electron $g$-factors. The $g$ factor of bound electrons is determined from ODMR studies [24].

In this paper we present a systematic theoretical study of the SDR in the threeand two-charge-state models with a focus concentrated on the conduction photoelectron spin polarization and circular polarization of edge PL induced by circularly-polarized radiation. It is shown that the two-charge-state theory is compatible with available experimental data on GaAsN alloys. The influence and specific role of each model parameters is analyzed and a complete set of them is determined for a particular GaAsN alloy sample. The experimental curves are compared not only with the results of exact model calculation but also with approximate analytical equations derived in the limit of weak or strong pumping powers and small or high magnetic fields.

The paper is organized as follows. In section 2 we extend the theory of Shockley-Read recombination to allow for the spin polarization and spin-dependent recombination of photocarriers. We start from a more general three-charge-state model of the SDR and then reduce the consideration to the two-charge-state model for the particular case of $\mathrm{cw}$ photoexcitation. The exact equations are derived for the PL intensity and circular polarization, and analytical equations are derived in the simple limiting cases. In section 3 , the values of nine parameters of the two-charge-state model are estimated by using available experimental data and reasonable theoretical considerations. Experimental and theoretical dependencies of the SDR ratio and degree of PL circular polarization on the pumping intensity and magnetic field are presented, compared and discussed in section 4. Section 5 contains the concluding remarks.

\section{Theory}

\subsection{Three-charge-state model}

In solids, impurity atoms can form several energy levels in the band gap. To illustrate we refer to four-level Au impurity in germanium [27] and three-charged states of interstitial boron in silicon [28] or Fe, Rh in photorefractive crystals [29, 30]. The multicharged states of $\mathrm{Ga}$ interstitials in GaAs are analyzed in reference [31], see also references therein. The statistics of defects with several trapping levels in semiconductors was analyzed decades ago [32. Until now the rate equations describing light-induced changes in electron and hole densities and occupation of three-charged impurity states has been treated for spin-unpolarized charge carriers [29, 30]. In this section we generalize the consideration to the spin polarized photoelectrons and their spin-dependent recombination via deep paramagnetic centers.

The three-charge-state model is summarized in figure 1(a). We assume that each 

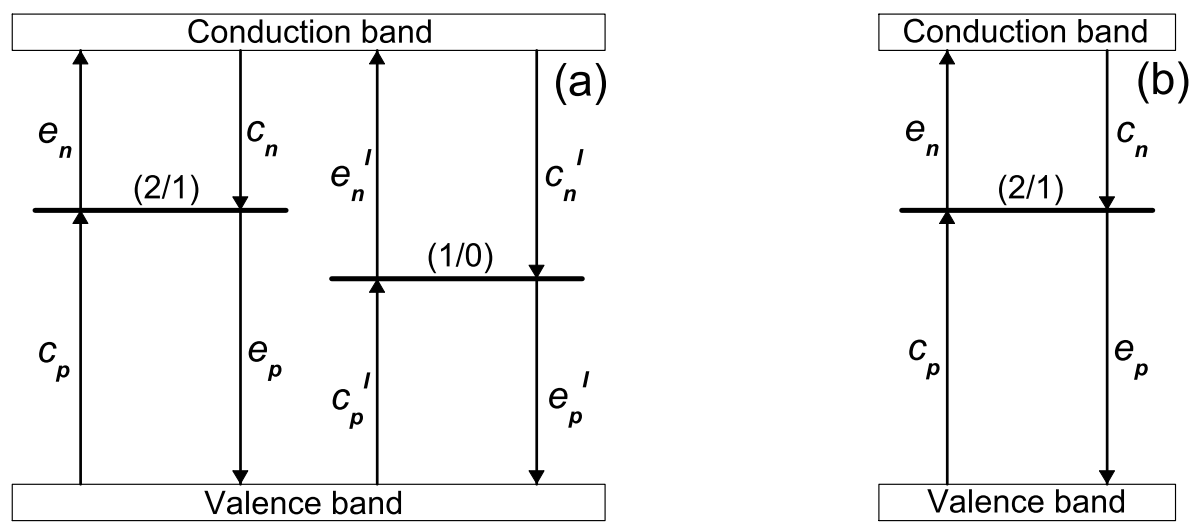

Figure 1. Band diagrams of the three-charge-state (a) and two-charge-state (b) models. Upper arrows indicate the excitation (up arrows) and recombination (down arrows) of conduction electrons described by the coefficients $e_{n}, c_{n}$ for the $(2 / 1)$ transition between the two- and one-electron defect states and $e_{n}^{\prime}, c_{n}^{\prime}$ for the $(1 / 0)$ transition. Lower arrows illustrate the hole excitation $\left(e_{p}, e_{p}^{\prime}\right)$ and recombination $\left(c_{p}, c_{p}^{\prime}\right)$.

deep center can be in one of three states $D_{i}(i=0,1,2)$ representing the center containing, respectively, no electrons, one bound electron with uncompensated spin $\pm 1 / 2$ and two bound electrons in the singlet state with the zero total spin. The corresponding densities are denoted as $N_{0}, N_{1}, N_{2}$ and satisfy the condition

$$
N_{0}+N_{1}+N_{2}=N_{c},
$$

where $N_{c}$ is the total density of the deep-level centers.

Under normal incidence of the circularly polarized light in the absence of a magnetic field, the electronic spins are polarized along the excitation direction (the axis $z$ ). Let us introduce the notations $n_{ \pm}, N_{ \pm}$, respectively, for the concentration of the free electrons and paramagnetic centers with the electron-spin component $\pm 1 / 2$ of the electron spin. Note that $N_{+}+N_{-}=N_{1}$. Hereafter, we assume the spin relaxation of photoholes to be very fast, neglect their spin polarization and use the notation $p$ for the hole density. Taking into account interband optical generation of free carriers, their recombination and thermal ionization from impurity levels, the rate equations for the electron densities $n_{ \pm}$, the hole density $p$ and the densities $N_{ \pm}, N_{0}$ at zero magnetic field are

$$
\begin{aligned}
& \frac{d n_{ \pm}}{d t}+\frac{n_{ \pm}-n_{\mp}}{2 \tau_{s}}+\gamma n_{ \pm} p=-R_{n, \pm}+G_{n, \pm}-R_{n, \pm}^{\prime}+G_{n, \pm}^{\prime}+G_{ \pm}^{\mathrm{opt}}, \\
& \frac{d N_{ \pm}}{d t}+\frac{N_{ \pm}-N_{\mp}}{2 \tau_{s c}}= \\
& -R_{n, \mp}+G_{n, \pm}+R_{n, \pm}^{\prime}-G_{n, \pm}^{\prime}+R_{p, \pm}-G_{p, \pm}-R_{p, \pm}^{\prime}+G_{p, \pm}^{\prime}, \\
& \frac{d p}{d t}+\gamma n_{ \pm} p=-R_{p}-R_{p}^{\prime}+G_{p}+G_{p}^{\prime}+G^{\mathrm{opt}} \\
& \frac{d N_{0}}{d t}=-R_{n}^{\prime}+G_{n}^{\prime}+R_{p}^{\prime}-G_{p}^{\prime} .
\end{aligned}
$$


Here $G_{ \pm}^{\text {opt }}$ are the photogeneration rates of free electrons with the spin $\pm 1 / 2, G^{\text {opt }}$ is the total generation rate $G_{+}^{\text {opt }}+G_{-}^{\text {opt }}$ proportional to the incident light intensity $J, \gamma$ is the coefficient for band-to-band recombination, the times $\tau_{s}$ and $\tau_{s c}$ describe the spin relaxation of free and single bound electrons (apparently, at room temperature $\tau_{s} \ll \tau_{s c}$ ). The rates for electron or hole recombination and thermal excitation are given by

$$
\begin{aligned}
& R_{n, \pm}=2 c_{n} N_{\mp} n_{ \pm}, R_{n, \pm}^{\prime}=c_{n}^{\prime} N_{0} n_{ \pm}, \\
& R_{n}^{\prime}=R_{n,+}^{\prime}+R_{n,-}^{\prime}=c_{n}^{\prime} N_{0}\left(n_{+}+n_{-}\right), \\
& R_{p}=2 R_{p, \pm}=c_{p} N_{2} p, R_{p, \pm}^{\prime}=c_{p}^{\prime} N_{ \pm} p, R_{p}^{\prime}=c_{p}^{\prime}\left(N_{+}+N_{-}\right) p, \\
& G_{n, \pm}=\frac{e_{n}}{2} N_{2}, G_{n, \pm}^{\prime}=e_{n}^{\prime} N_{ \pm}, G_{n}^{\prime}=G_{n+}^{\prime}+G_{n-}^{\prime}, \\
& G_{p, \pm}=e_{p} N_{ \pm}, G_{p}=e_{p}\left(N_{+}+N_{-}\right), G_{p}^{\prime}=2 G_{p, \pm}^{\prime}=e_{p}^{\prime} N_{0},
\end{aligned}
$$

the coefficients $c_{n}, e_{n}, c_{p}, e_{p}$ describe the $(2 / 1)$ processes $D_{1}+e^{-} \leftrightarrow D_{2}$ and $D_{2}+h^{+} \leftrightarrow D_{1}$ and have the same meaning as the corresponding coefficients in the original article by Shockley and Read [33] (see also the book [34]), the similar coefficients $c_{n}^{\prime}, e_{n}^{\prime}, c_{p}^{\prime}, e_{p}^{\prime}$ refer to the $(1 / 0)$ processes $D_{0}+e^{-} \leftrightarrow D_{1}$ and $D_{1}+h^{+} \leftrightarrow D_{0}$, where $e^{-}$and $h^{+}$symbolize an electron and a hole.

In addition to equation (1) the densities in consideration satisfy the neutrality equation

$$
p=n+N_{2}-N_{0}-\left(N_{2}^{0}-N_{0}^{0}\right),
$$

where $N_{j}^{0}$ are the equilibrium densities given by 32

$$
\frac{N_{2}^{0}}{N_{1}^{0}}=\frac{1}{2} \exp \left(\frac{\mu-E(2 / 1)}{k_{B} T}\right), \quad \frac{N_{1}^{0}}{N_{0}^{0}}=2 \exp \left(\frac{\mu-E(1 / 0)}{k_{B} T}\right)
$$

and $N_{0}^{0}+N_{1}^{0}+N_{2}^{0}=N_{c}$, where $\mu$ is the electron equilibrium chemical potential, $E(i+1 / i)$ is the change in energy associated with adding one electron to the center in the process $D_{i}+e^{-} \rightarrow D_{i+1}$ and the factors 2 and $1 / 2$ take into account the double spin degeneracy of the paramagnetic state.

The set of equations (11)-(3) contains a lot of parameters. To simplify the analysis we assume that the photoexcitation rate $G^{\text {opt }}$ is high enough to neglect the thermal excitation rates $G_{n, \pm}, G_{n, \pm}^{\prime}, G_{p}$ and $G_{p}^{\prime}$. Moreover, we neglect the influence of band-toband recombination on the kinetics of charge carriers, set $\gamma$ to zero in equations (2) and use the thus obtained values of $n_{+}, n_{-}$and $p$ to calculate the photoluminescence (PL) intensity and polarization. For the sake of distinctness, we also assume that, in equilibrium, all the centers bind one electron per center, i.e., are paramagnetic and $N_{1}^{0}=N_{c}$.

Instead of four variables $n_{ \pm}, N_{ \pm}$we introduce the total free-electron density $n=n_{+}+n_{-}$and $\operatorname{spin} S_{z}=\left(n_{+}-n_{-}\right) / 2$, the density of paramagnetic centers (or centers with uncompensated spins) $N_{1}=N_{+}+N_{-}$and the bound-electron total spin $S_{c z}=\left(N_{+}-N_{-}\right) / 2$. In a transverse magnetic field the electron spin exhibits the Larmor precession and, instead of the scalars $S_{z}$ and $S_{c z}$, one should define the pseudovectors $\boldsymbol{S}$ 
and $\boldsymbol{S}_{c}$ pointing in the directions of the average free- and bound-electron spins. Then the set of kinetic equations (2) in the presence of magnetic field are rewritten as

$$
\begin{aligned}
& \frac{d n}{d t}+c_{n}\left(N_{1} n-4 \boldsymbol{S}_{c}\right)+c_{n}^{\prime} N_{0} n=G^{\mathrm{opt}}, \\
& \frac{d p}{d t}+\left(c_{p} N_{2}+c_{p}^{\prime} N_{1}\right) p=G^{\mathrm{opt}}, \\
& \frac{d \boldsymbol{S}}{d t}+c_{n}\left(N_{1} \boldsymbol{S}-\boldsymbol{S}_{c} n\right)+\boldsymbol{S}\left(\frac{1}{\tau_{s}}+c_{n}^{\prime} N_{0}\right)+\boldsymbol{S} \times \boldsymbol{\omega}=\frac{P_{i}}{2} G^{\mathrm{opt}} \boldsymbol{o}_{z}, \\
& \frac{d \boldsymbol{S}_{c}}{d t}+c_{n}\left(\boldsymbol{S}_{c} n-N_{1} \boldsymbol{S}\right)+\frac{\boldsymbol{S}_{c}}{\tau_{s c}}-c_{n}^{\prime} N_{0} \boldsymbol{S}+c_{p}^{\prime} p \boldsymbol{S}_{c}+\boldsymbol{S}_{c} \times \boldsymbol{\Omega}=0, \\
& \frac{d N_{0}}{d t}+c_{n}^{\prime} N_{0} n+c_{p}^{\prime} N_{1} p=0 .
\end{aligned}
$$

Here $P_{i}=\left(G_{+}^{\mathrm{opt}}-G_{-}^{\mathrm{opt}}\right) / G^{\mathrm{opt}}, \boldsymbol{o}_{z}$ is the unit vector directed along the normal $z$ coinciding with the exciting-light propagation direction, $\boldsymbol{\omega}$ and $\boldsymbol{\Omega}$ are the Larmor frequencies defined by $\hbar \boldsymbol{\omega}=g \mu_{B} \boldsymbol{B}, \hbar \boldsymbol{\Omega}=g_{c} \mu_{B} \boldsymbol{B}, g$ and $g_{c}$ are the $g$-factors of free and bound electrons, $\boldsymbol{B}$ is the external magnetic field and $\mu_{B}$ is the Bohr magneton. In the following

we assume $\boldsymbol{B} \| y \perp z$. Equations (4a) -(4e) together with equations (1) and (3) form a complete set in the proposed model.

\subsection{Continuous wave photoexcitation}

Under continuous wave photoexcitation the time derivatives vanish and the equations for $\boldsymbol{S}$ and $\boldsymbol{S}_{c}$ are reduced to

$$
\begin{aligned}
& \frac{\boldsymbol{S}}{T}-\frac{\boldsymbol{S}_{c}}{\tau_{c}}+\boldsymbol{S} \times \boldsymbol{\omega}=\frac{P_{i}}{2} G^{\mathrm{opt}} \boldsymbol{o}_{z}, \\
& \frac{\boldsymbol{S}_{c}}{T_{c}}-\frac{\boldsymbol{S}}{\tau}+\boldsymbol{S}_{c} \times \boldsymbol{\Omega}=0,
\end{aligned}
$$

where the four introduced times are defined by

$$
\begin{array}{lll}
\frac{1}{T}=\frac{1}{\tau_{s}}+\frac{1}{\tau}, & \frac{1}{\tau_{c}}=c_{n} n, \\
\frac{1}{T_{c}}=\frac{1}{\tau_{s c}}+c_{n} n+c_{p}^{\prime} p, & \frac{1}{\tau}=c_{n} N_{1}+c_{n}^{\prime} N_{0} .
\end{array}
$$

By using equations (5) we can present the free- and bound-electron spin components $S_{z}, S_{c z}$ in the form

$$
S_{z}=\frac{P_{i} G^{\mathrm{opt}} T}{2 \Psi}, \quad S_{c z}=\Lambda S_{z} .
$$

where

$$
\begin{aligned}
& \Psi=1+\omega^{2} \tilde{T}^{2}-\eta \frac{\left(1-\omega \Omega \tilde{T} \tilde{T}_{c}\right)^{2}}{1+\Omega^{2} \tilde{T}_{c}^{2}}, \\
& \Lambda=\frac{T_{c}}{\tau} \frac{1-\omega \Omega \tilde{T} \tilde{T}_{c}}{1+\Omega^{2} \tilde{T}_{c}^{2}},
\end{aligned}
$$


and $\eta=\left(T T_{c} / \tau \tau_{c}\right), \quad \tilde{T}=T / \sqrt{1-\eta}, \quad \tilde{T}_{c}=T_{c} / \sqrt{1-\eta}$. The transverse spin components $S_{x}, S_{c x}($ for $\boldsymbol{B} \| y)$ are given by

$$
S_{x}=\frac{T}{\tau_{c}} \frac{\omega \tau_{c} S_{z}+\Omega T_{c} S_{c z}}{1-\eta}, \quad S_{c x}=\frac{T_{c}}{\tau} \frac{\omega T S_{z}+\Omega \tau S_{c z}}{1-\eta} .
$$

The densities $p, N_{0}, N_{2}$ can be expressed via $n$ and $N_{1}$ as

$$
\begin{aligned}
& p=\frac{c_{n}^{\prime} n}{2 c_{p}^{\prime} N_{1}} \frac{N_{c}-N_{1}+n}{1+\left(c_{n}^{\prime} / 2 c_{p}^{\prime}\right)\left(n / N_{1}\right)}, \\
& N_{0}=\frac{c_{p}^{\prime} N_{1}}{c_{n}^{\prime} n} p=\frac{1}{2} \frac{N_{c}-N_{1}+n}{1+\left(c_{n}^{\prime} / 2 c_{p}^{\prime}\right)\left(n / N_{1}\right)}, \\
& N_{2}=\frac{1}{2} \frac{\left(N_{c}-N_{1}\right)\left[1+\left(c_{n}^{\prime} / c_{p}^{\prime}\right)\left(n / N_{1}\right)\right]-n}{1+\left(c_{n}^{\prime} / 2 c_{p}^{\prime}\right)\left(n / N_{1}\right)} .
\end{aligned}
$$

Excluding $p, N_{0}, N_{2}, \boldsymbol{S}$ and $\boldsymbol{S}_{c}$ from the complete set of equations we obtain, instead of equations (4a) and (4b), the following two coupled nonlinear equations for $n$ and $N_{1}$

$$
\begin{gathered}
\frac{1}{2}\left\{c_{p} \frac{\left(N_{c}-N_{1}\right)\left[1+\left(c_{n}^{\prime} / c_{p}^{\prime}\right)\left(n / N_{1}\right)\right]-n}{1+\left(c_{n}^{\prime} / 2 c_{p}^{\prime}\right)\left(n / N_{1}\right)}+2 c_{p}^{\prime} N_{1}\right\} \\
\times \frac{N_{c}-N_{1}+n}{\left(2 c_{p}^{\prime} / c_{n}^{\prime}\right)\left(N_{1} / n\right)+1}=G^{\mathrm{opt}}, \\
c_{n} n N_{1}(1-\Sigma)+c_{n}^{\prime} N_{0}\left(n, N_{1}\right) n=G^{\mathrm{opt}},
\end{gathered}
$$

where

$$
\begin{aligned}
\Sigma= & \frac{4 \boldsymbol{S S}_{c}}{n N_{1}}=\frac{\left(P_{i} G^{\mathrm{opt}} T\right)^{2}}{n N_{1} \Psi^{2}} \\
& \times\left\{\Lambda+\frac{\eta}{(1-\eta)^{2}}\left[\omega^{2} \tau_{c} T+\Lambda \omega \Omega \tau \tau_{c}(1+\eta)+\Lambda^{2} \Omega^{2} \tau T_{c}\right]\right\} .
\end{aligned}
$$

According to equation (7) the degree of free-electron spin polarization is given by

$$
P=\frac{2 S_{z}}{n}=\frac{P_{i} G^{\mathrm{opt}} T}{n \Psi} .
$$

The intensity $I$ and degree of circular polarization of the photoluminescence $\rho$ can be found from

$$
I=I^{+}+I^{-} \propto n p, \quad \rho=\frac{I^{+}-I^{-}}{I}=P^{\prime} P
$$

where $I^{ \pm}$are the intensities of the $\sigma^{ \pm}$circularly-polarized components of the interband $\mathrm{PL}$, and $P^{\prime}=P_{1}^{\prime} P_{2}^{\prime}$, with the factor $P_{1}^{\prime}$ taking into account the selection rules for the room-temperature recombination of the conduction electrons with heavy and light holes, and $P_{2}^{\prime} \leq 1$ being the depolarizing factor arising due to possible multiple reflections from the sample boundaries [35].

\subsection{Two-charge-state model}

As follows from references [13, 15, 16, 17, 18, 24, 36] and demonstrated below the SDR model taking into account only the deep-center states $D_{1}$ and $D_{2}$ satisfactorily describes 
the experiments on spin dynamics in GaAsN samples. Therefore, in what follows we focus on this particular model setting $c_{p}^{\prime}=0$ in which case $N_{0}=0, N_{c}=N_{1}+N_{2}$, $p=n+N_{c}-N_{1}$ and equations (12), (13) reduce to

$$
c_{p}\left(N_{c}-N_{1}\right)\left(N_{c}-N_{1}+n\right)=G^{\mathrm{opt}}, \quad c_{n} n N_{1}(1-\Sigma)=G^{\mathrm{opt}} .
$$

Optical orientation of electron spins at zero magnetic field. Let us consider particular limiting cases allowing for simple analytical expressions. In the absence of zero magnetic field the Larmor frequencies $\omega, \Omega$ vanish and the expressions for $S_{z}$ and $\Sigma$ reduce to

$$
S_{z}=\frac{P_{i} G^{\mathrm{opt}} T}{2(1-\eta)}, \quad \Sigma=\frac{\left(P_{i} G^{\mathrm{opt}} T\right)^{2}}{n N_{1}} \frac{T_{c}}{\tau} \frac{1}{(1-\eta)^{2}} .
$$

It is convenient to introduce the dimensionless variables

$$
X=\frac{G^{\mathrm{opt}}}{c_{p} N_{c}^{2}}, \quad Y=\frac{N_{c}-N_{1}}{N_{c}}=\frac{N_{2}}{N_{c}}, \quad Z=\frac{n}{N_{c}} .
$$

In terms of these variables one has

$$
\begin{array}{ll}
p & =N_{c}(Y+Z), \quad I \propto Z(Y+Z), \quad \rho=\frac{P^{\prime} P_{i}}{1-\eta} \frac{T}{\tau_{h}^{*}} \frac{X}{Z}, \\
\Lambda & =\frac{T_{c}}{\tau}, \quad \Psi=1-\eta, \\
\frac{1}{\tau} & =\frac{1-Y}{\tau^{*}}, \quad \frac{1}{T}=\frac{1}{\tau_{s}}+\frac{1-Y}{\tau^{*}}, \\
\frac{1}{\tau_{c}} & =\frac{Z}{\tau^{*}}, \quad \frac{1}{T_{c}}=\frac{1}{\tau_{s c}}+\frac{Z}{\tau^{*}},
\end{array}
$$

where $\tau^{*}=\left(c_{n} N_{c}\right)^{-1}$ and $\tau_{h}^{*}=\left(c_{p} N_{c}\right)^{-1}$.

According to equations (13) and (16) the variables $Y$ and $Z$ satisfy the equations

$$
\begin{aligned}
& Y(Y+Z)=X, \\
& \frac{1-Y}{a}\left\{Z-P_{i}^{2}\left(\frac{\tau_{s}}{\tau_{h}^{*}}\right)^{2} X^{2} \frac{Z+\tau^{*} / \tau_{s c}}{\left[Z+\tau^{*} / \tau_{s c}+(1-Y) \tau_{c} / \tau_{s c}\right]^{2}}\right\}=X,
\end{aligned}
$$

where $a=c_{p} / c_{n}$.

In the low-power limit we have $\tau_{c} \rightarrow \infty, \eta \rightarrow 0$ and

$$
\begin{aligned}
& n=\tau^{*} G^{\mathrm{opt}}, \quad \rho=P^{\prime} P_{i} \frac{\tau_{s}}{\tau_{s}+\tau^{*}}, \\
& p \propto N_{c}-N_{1} \propto \sqrt{G^{\mathrm{opt}}}, \quad I \propto\left(G^{\mathrm{opt}}\right)^{3 / 2} .
\end{aligned}
$$

The first nonvanishing corrections to $n$ and $\rho$ are taken into account in equation (21) by the replacement $\tau^{*} \rightarrow \tau^{*}\left(1+\sqrt{\tau_{h}^{*} G^{\text {opt }} / N_{c}}\right)$. In particular, this means that

$$
\rho=P^{\prime} P_{i} \frac{\tau_{s}}{\tau_{s}+\tau^{*}}\left(1-\frac{\tau^{*}}{\tau_{s}+\tau^{*}} \sqrt{\frac{\tau_{h}^{*} G^{\mathrm{opt}}}{N_{c}}}\right) .
$$

However, as soon as a value of $\left(\tau_{s} \tau_{s c} / \tau^{*} \tau_{h}^{*}\right) \sqrt{X}$ exceeds unity the main correction to $\rho$ originates from the spin-dependent recombination, it is linear in $G^{\text {opt }}$ and given by

$$
\rho=P^{\prime} P_{i} \frac{\tau_{s}}{\tau_{s}+\tau^{*}}\left(1+\frac{\tau_{s}}{\tau_{s}+\tau^{*}} \frac{\tau_{s c} G^{\mathrm{opt}}}{N_{c}}\right) .
$$


With increasing pump intensity, $Y$ monotonously increases and saturates up to some limiting value controlled by the ratio $c_{p} / c_{n}$. This limiting value $Y_{\infty} \equiv Y\left(G^{\text {opt }} \rightarrow \infty\right)$ satisfies a third-order algebraic equation

$$
\left(1-Y_{\infty}\right)\left(1-b Y_{\infty}^{2}\right)-a Y_{\infty}=0
$$

where $b=P_{i}^{2}\left(\tau_{s} / \tau_{h}^{*}\right)^{2}$. Note that according to equation (20) one has asymptotically $Z\left(G^{\text {opt }} \rightarrow \infty\right) \rightarrow X / Y_{\infty}$. If $a=c_{p} / c_{n} \ll|1-\sqrt{b}|$, then

$$
Y_{\infty} \approx\left\{\begin{array}{cl}
1-\frac{a}{1-b} & \text { for } b<1 \\
\frac{1}{\sqrt{b}}\left(1-\frac{a}{2(\sqrt{b}-1)}\right) & \text { for } b>1 .
\end{array}\right.
$$

For $b \approx 1$ and $a \ll 1$, one has $Y_{\infty}=1-\sqrt{a / 2}$.

Hanle effect at transverse magnetic field. Assuming $T \ll T_{c}$ and comparable values of $g$ and $g_{c}$ we can separately analyze two regions of the transverse magnetic field: (I) the low-field region where $\omega T \ll 1$ and the product $\Omega T_{c}$ is arbitrary, and (II) the high-field region where $\Omega T \gg 1$ and the product $\omega T$ is arbitrary.

In the region I, at low pumping level, equation (23) derived at $B=0$ transfers to

$$
\rho(B)=P^{\prime} P_{i} \frac{\tau_{s}}{\tau_{s}+\tau^{*}}\left(1+\frac{\tau_{s}}{\tau_{s}+\tau^{*}} \frac{\tau_{s c} G^{\text {opt }}}{N_{c}} \frac{1}{1+\Omega^{2} T_{c}^{2}}\right) .
$$

One can see that in this particular case $\rho(B)$ is a sum of a constant and a weak narrow Lorentzian.

In the region II, at arbitrary pumping level, one can neglect the influence of bound electrons upon the formation of the free-electron density and polarization, set $\Sigma$ to zero and use the equations

$$
n=\tau G^{\mathrm{opt}}, \quad \rho(B)=P^{\prime} P_{i} \frac{\tau_{s}}{\tau_{s}+\tau} \frac{1}{1+\omega^{2} T^{2}}=\frac{\rho_{0}}{1+\left(B / B_{1 / 2}\right)^{2}},
$$

where $\rho_{0}=P^{\prime} P_{i} \tau_{s} /\left(\tau_{s}+\tau\right)$ and $B_{1 / 2}=\hbar /\left(g \mu_{B} T\right)$ is the half-width at half-maximum of the Lorentzian. At weak incident-light intensities, the lifetime $\tau$ can be replace by the characteristic time $\tau^{*}$.

Electron density for unpolarized carriers. The system is spin-unpolarized under linearly-polarized photoexcitation as well as for $\sigma^{ \pm}$pumping in the limit of very strong transverse magnetic fields. In the both cases, in order to find $n$ and $N_{1}$ one can formally set $P_{i}=0$ and reduce a pair of equations (20) to

$$
\frac{Y^{2}(1-Y)}{1-(1+a) Y}=X
$$

To trace the saturation of $Y$ and asymptotic behavior of $Z$ with the increasing pump intensity, we set $X \rightarrow \infty$ in equation (28) and obtain

$$
\begin{aligned}
& Y_{\infty} \approx \frac{1}{1+a}\left(1-\frac{a}{(1+a)^{3}} \frac{N_{c}}{\tau_{h}^{*} G^{\mathrm{opt}}}\right), \\
& Z\left(G^{\mathrm{opt}} \rightarrow \infty\right) \approx \frac{(1+a) \tau_{h}^{*} G^{\mathrm{opt}}}{N_{c}}-\frac{1}{(1+a)^{2}} .
\end{aligned}
$$




\section{Selection of model parameter values}

The two-charge-state model is characterized by two free (controllable) variables, the total generation rate $G^{\text {opt }}$ and the strength of magnetic field $B$, and nine fitting parameters, namely, Landé factors $g$ and $g_{c}$, the spin relaxation times $\tau_{s}$ and $\tau_{s c}$, the recombination times $\tau^{*}=\left(c_{n} N_{c}\right)^{-1}$ and $\tau_{h}^{*}=\left(c_{p} N_{c}\right)^{-1}$, the degrees of polarization $P_{i}, P^{\prime}$ and the density of deep centers $N_{c}$. In the present section we will estimate values of these parameters by using available experimental data and reasonable theoretical considerations.

\section{Lande g-factors of free and bound electrons}

The recent studies 21, 24 of ODMR have provided unambiguous experimental evidence that, in dilute nitrides $\mathrm{GaAs}_{1-x} \mathrm{~N}_{x}$ and $\mathrm{Ga}_{1-y} \mathrm{In}_{y} \mathrm{As}_{1-x} \mathrm{~N}_{x}$ with a few percent of nitrogen $(x \leq 3.3 \%)$, the deep paramagnetic centers are formed by $\mathrm{Ga}_{i}$ self-interstitials. In the above notations, the defect state $D_{1}$ occupied by a single electron represents the doubly positive charged level $\mathrm{Ga}_{i}^{2+}$ while the singlet $D_{2}$ occupied by a pair of electrons is identified as the singly positive state $\mathrm{Ga}_{i}^{1+}$. The bound-electron $g$ factor $g_{c}$ determined from the ODMR measurements has been found to be very close to 2 . The positive sign of $g_{c}$ has been independently determined from asymmetry in the depolarization of edge photoluminescence in a transverse magnetic field (Hanle effect) at oblique incidence of the exciting radiation and oblique-angle detection of the luminescence [13, 37. The results of references [13, 21, 24, 37] are in agreement with the existing expectation that the $g$ factors of electrons bound to deep levels, as a rule, exhibit only a small renormalization with respect to the gyromagnetic factor $g_{0}=2.0023$ of a free electron in vacuum.

On the other hand, the $g$ values of free carriers, electrons and holes, in semiconductors with small and moderate band gaps can drastically differ from $g_{0}$. Particularly, for conduction-band electrons in bulk GaAs the $g$ factor equals -0.44 at helium temperature. In $\mathrm{GaAs}_{1-x} \mathrm{~N}_{x}$ with a few percent of $\mathrm{N}$ the value of electron $g$ factor shifts upwards. The experimental study [38, 39] of the Zeeman splitting of $\sigma^{+}$and $\sigma^{-}$circularly polarized components of the photoluminescence spectra of $\operatorname{GaAs}_{1-x} \mathrm{~N}_{x}(x \leq 0.6 \%)$ have shown that the conduction-electron $g$ factor exhibits a sign reversal from negative to positive at $x \approx 0.04 \%$ and increases abruptly up to 0.7 in a very narrow compositional window between $x=0.04 \%$ and $x=0.1 \%$. For $0.1 \%<x \leq 0.6 \%$, the dependence $g(x)$ has a not well-defined behaviour and fluctuates around 0.7 for the highest $x$ values. The striking difference from the value -0.44 is attributed to the resonant defect level in the conduction band introduced by nitrogen and the repulsion between the GaAs host matrix conduction band edge and a higher lying band of localized $\mathrm{N}$ resonant states (the so-called band-anticrossing model introduced by Shan, Walukiewicz et al [40, see also [41]). The agreement between experimental data and theoretical calculation is remarkably improved in the generalized band-anticrossing model which takes into account the interaction of the conduction-band minimum with localized resonant states formed by nitrogen-atom pairs and clusters [38, 39]. 
The positive sign of $g$ in $\mathrm{GaAs}_{1-x} \mathrm{~N}_{x}$ film with $x=2.1 \%$ was found at room temperature from the Hanle effect asymmetry under oblique excitation and detection [37] while its absolute value $|g| \approx 0.9$ follows from the electron spin quantum beats in photoluminescence recorded in a transverse magnetic field after the pulse excitation [16]. The data are in agreement with the value $g \approx+1$ estimated in reference [37] from the relationship between $g$ and electron effective mass $m^{*} \approx 0.14 m_{0}$ measured for $x=2 \%$ in reference 42 . The positive free-electron $g$-factor $g=0.97$ was measured in $\mathrm{GaAs}_{1-x} \mathrm{~N}_{x} /$ GaAs quantum well with $x=1.5 \%$ by time resolved Kerr rotation at $220 \mathrm{~K}[43$. On the other hand, in reference [44] only a slow decrease of $|g|$ from 0.28 to 0.22 for $x$ increasing from $0.09 \%$ to $0.9 \%$ has recently been reported from time-resolved Kerr rotation measurements in $\mathrm{GaAs}_{1-x} \mathrm{~N}_{x}$ films at room temperature. The difference with the above values may be related to variations in nitrogen-atom spatial distribution inside dilute nitride samples studied by the two groups.

Below, we will use $g=+1, g_{c}=+2$.

\section{Characteristic free electron and hole lifetimes $\tau^{*}$ and $\tau_{h}^{*}$}

According to equation (4b), in the two-charge-state model $\left(c_{p}^{\prime}=0\right)$ the hole recombination rate equals $c_{p} N_{2} p$. In the limit of high pump intensities, $G^{\text {opt }} \rightarrow \infty$, the hole lifetime $\tau_{h} \equiv\left(c_{p} N_{2}\right)^{-1}$ tends to a limit of $\tau_{h}^{*}=\left(c_{p} N_{c}\right)^{-1}$. The regime of high pumping was realized by Kalevich et al [16] under pulsed optical excitation of $\mathrm{GaAs}_{0.979} \mathrm{~N}_{0.021}$ alloy at room temperature. The ratio of the photoelectron density $n$ and $N_{c}$ exceeded 10 and the photoluminescence decay time at the initial stage, (15.0 $\pm 0.5) \mathrm{ps}$, could be identified with $\tau_{h}^{*} / 2$. Thus, in this particular sample, the time $\tau_{h}^{*}$ can be taken equal to 30 ps or a little bit shorter because, as the analysis shows, in the experimental conditions a maximum value of $N_{2}$ was slightly smaller than $N_{c}$.

In the low intensity limit, where $\tau \rightarrow \tau^{*}$, one can use equations (21). Moreover, it follows from the experiment [16] that the three characteristic times $\tau^{*}, \tau_{h}^{*}$ and $\tau_{s}$ satisfy the hierarchy: $\tau^{*} \ll \tau_{h}^{*} \ll \tau_{s}$. Therefore, at low pumping levels the lifetime, $T$, of free-electron spin polarization given by the first equation (6) is close to $\tau^{*}$. This allowed us to determine $\tau^{*}$ from measurements of the Hanle effect at low incident light intensity $J$. The interpolation of the experimental dependence $B_{1 / 2}(J) \approx \hbar /\left(g \mu_{B} \tau\right)$ of the freeelectron Hanle curve half-width to the limit $J \rightarrow 0$ yields [36], for $g=1$, the conduction electron lifetime $\tau^{*}=\tau(J \rightarrow 0)=1.9$ ps.

For the model calculations we will use $\tau^{*}=1.9 \mathrm{ps}$ and $\tau_{h}^{*}=27 \mathrm{ps}$.

\section{Spin relaxation times of free and bound electrons}

The spin relaxation time $\tau_{s}$ of free photoelectrons was found [15, 16] from the decay curves of

$$
S_{z}(t) \propto \frac{I^{+}(t)-I^{-}(t)}{p(t)} \propto \frac{I^{+}(t)-I^{-}(t)}{\sqrt{I(t)}}
$$

and, independently, from the PL intensity decay, $I(t)$, at the second stage of the spin-dependent electron dynamics, the partial intensities $I^{ \pm}$being introduced in equation (15). The obtained two values $(150 \pm 15)$ ps and $(144 \pm 4)$ ps, respectively, 
coincide within the uncertainty limits, confirming the interpretation of the experimental data. The spin-Kerr rotation measurements 44 performed on three GaAsN samples at room temperature give for the free-electron spin lifetime $T$ the values of 60,120 and $125 \mathrm{ps}$ which can serve as a lower bound for the spin relaxation time $\tau_{s}$ in these samples in agreement with the above two values of $\tau_{s}$.

The spin-relaxation time of bound electrons, $\tau_{s c}$, can be deduced from measurements of the Hanle depolarization curve [13, 36], or, to be more precise, the narrow part of this curve related to the depolarization of bound electrons. Indeed, according to equation (26) the half-width of the narrow Lorentzian is given by $B_{1 / 2}^{c}=\hbar / g_{c} \mu_{B} T_{c}$ which reduces, at $J \rightarrow 0$, to $\hbar / g_{c} \mu_{B} \tau_{s c}$. By using the values $B_{1 / 2}^{c} \approx 85 \mathrm{G}$ and $g_{c}=+2$, we obtained [36] $\tau_{s c} \approx 700 \mathrm{ps}$.

Later on we will use $\tau_{s}=140 \mathrm{ps}, \tau_{s c}=700 \mathrm{ps}$.

4. Initial photoelectron spin polarization $P_{i}$ and the factor $P^{\prime}$ relating $\rho$ with $P$

The determination of the initial spin polarization $P_{i}$ from experiments on polarized photoluminescence can be complicated by the presence of a small additional contribution of unclear origin to the secondary emission. For the circularly-polarized incident light, this contribution is also polarized and its circular polarization is insensitive to the transverse magnetic field up to very high fields. Taking into account the additional contribution the measured degree of circular polarization is presented as $\rho_{\exp }=(1-j) \rho+j \rho_{\text {add }}$, where $\rho$ is given by equation (15), $\rho_{\text {add }}$ is the polarization of the additional radiation and $j$ is its fraction in the total intensity of the secondary radiation. Assuming $j$ to be small, we can approximate $\rho_{\exp }$ to

$$
\rho_{\text {exp }}=\rho+\rho_{\text {res }}
$$

where $\rho_{\text {res }}=j \rho_{\text {add }}$ is the effective "residual" polarization. By using equation (30) one can find $P_{i}$ from the following equation

$$
P_{i} \approx \frac{\rho_{\exp }(B=5 \mathrm{kG})-\rho_{\text {res }}}{\rho_{\exp }(B=0, \text { high } J)-\rho_{\text {res }}} P(B=0 \text {, high } J) .
$$

Here $\rho_{\exp }(B=5 \mathrm{kG})$ is the degree of circular polarization measured at the moderate value of the transverse magnetic field, $B=5 \mathrm{kG}$, high enough in order to neglect the spin polarization of bound electrons and apply equation (27) and, on the other hand, low enough to satisfy the condition $\omega T \ll 1 ; \rho_{\exp }(B=0$, high $J)$ is the polarization degree measured at zero magnetic field and at a high photoexcitation level where the spin-filter effect results in almost $100 \%$ spin polarization of the free photoelectrons, $P(B=0$, high $J) \approx 1$. It follows then from equation (15) that

$$
P^{\prime}=\frac{\rho_{\exp }(B=0, \text { high } J)-\rho_{\text {res }}}{P(B=0, \text { high } J)} .
$$

Furthermore, according to equation (27), at the moderate magnetic field one has $\rho_{\exp }(B=5 \mathrm{kG})=P^{\prime} P_{i} \tau_{s} /\left(\tau+\tau_{s}\right)+\rho_{\text {res }}$. In the studied GaAsN alloys, at the moderate intensities, the free-electron lifetime is much shorter than their spin relaxation time and $\rho_{\exp }(B=5 \mathrm{kG})$ reduces to $P^{\prime} P_{i}+\rho_{\text {res }}$ which leads to $P_{i}=\left[\rho_{\exp }(B=5 \mathrm{kG})-\rho_{\text {res }}\right] / P^{\prime}$ and finally to equation (31). 


\section{Density of deep centers $N_{c}$}

The density of deep centers responsible for the spin-dependent recombination can be found by fitting the measured dependencies of the PL intensity and polarization on the incident light intensity with computation of the kinetic equations (4a) $-(4 e)$. The inhomogeneous terms in these equations are proportional to the generation rate $G^{\text {opt }}$ which can be expressed in terms of the excitation photon energy $E_{\mathrm{ph}}$, the incident light intensity $J$, the light absorption coefficient $\alpha$ and the beam spot area on the sample surface, $S^{*}$. Taking into account that the thickness of the GaAsN layer $L$ is smaller than $\alpha^{-1}$ and assuming that each photon creates one carrier pair we have

$$
G^{\mathrm{opt}}=\frac{\alpha J}{E_{\mathrm{ph}} S^{*}}
$$

Let us estimate a typical value of $G^{\text {opt }}$ in our experiments performed on GaAs $_{0.979} \mathrm{~N}_{0.021}$ at room temperature and the energy $E_{\mathrm{ph}}=1.312 \mathrm{eV}$ of the exciting photons. For this nitrogen content the room-temperature band gap of the alloy equals $E_{g} \approx 1.106 \mathrm{eV}\left[12\right.$, and, therefore, the excess photon energy $E_{\mathrm{ph}}-E_{g}=206 \mathrm{meV}$. We take $\alpha=2 \times 10^{4} \mathrm{~cm}^{-1}$ for this difference $E_{\mathrm{ph}}-E_{g}$ in accordance to values $2.4 \times 10^{4} \mathrm{~cm}^{-1}$ and $1.6 \times 10^{4} \mathrm{~cm}^{-1}$ measured in the alloys with $x=5 \%$ [45] and $x=2.3 \%$ [46] in the wide range of photon energies. For the light spot diameter $0.2 \mathrm{~mm}$ and the intensity $J=1 \mathrm{~mW}$ the generation rate $G^{\text {opt }}$ is $3 \times 10^{23} \mathrm{~cm}^{-3} \mathrm{~s}^{-1}$.

A lower estimate for the critical value of the generation rate $G_{\mathrm{cr} \text {, circ }}^{\mathrm{opt}}$ at which the spin filter effect becomes remarkable can be obtained by setting $\Sigma\left(G^{\text {opt }}\right)$ to unity. At zero magnetic field, small intensities, $T_{c} \approx \tau_{s c}$ and $N_{1} \approx N_{c}$ one has from equations (13), (17)

$$
\Sigma=\frac{\left(P_{i} G^{\mathrm{opt}} T\right)^{2}}{n N_{1}} \frac{T_{c}}{\tau(1-\eta)^{2}} \approx P_{i}^{2} \frac{G^{\mathrm{opt}} T^{2} T_{c}}{N_{c} \tau^{* 2}} \approx P_{i}^{2} \frac{G^{\mathrm{opt}} \tau_{s c}}{N_{c}}
$$

and, thus, the critical value of $G^{\text {opt }}$ is given by

$$
G_{\mathrm{cr}, \mathrm{circ}}^{\mathrm{opt}}=\frac{N_{c}}{P_{i}^{2} \tau_{s c}}
$$

It follows from experimental data presented in figure $2 \mathrm{~b}$ that the spin filter effect develops to full scale at $J=75 \mathrm{~mW}$, so that $G_{\text {cr, circ }}^{\text {opt }} \sim 3 \times 10^{25} \mathrm{~cm}^{-3} \mathrm{~s}^{-1}$. Taking $P_{i}=0.24$, $\tau_{s c}=700 \mathrm{ps}$ and $T=\tau^{*}$ (since $\tau_{s} \gg \tau^{*}$ ) we obtain from equation (34) the estimation $N_{c}=10^{15} \mathrm{~cm}^{-3}$. The best agreement with experiment, see the details below, is obtained for the density of deep paramagnetic centers $N_{c}=3 \times 10^{15} \mathrm{~cm}^{-3}$, in agreement with the crude estimation. It is worth to mention that, according to equation (28), the

critical generation rate $G_{\mathrm{cr} \text {, lin }}^{\mathrm{opt}}$ at which the system exhibits nonlinear behaviour under linearly polarized photoexcitation is given by the condition $X=1$ with $X$ defined by equation (18) or, equivalently, by

$$
G_{\mathrm{cr}, \operatorname{lin}}^{\mathrm{opt}}=\frac{N_{c}}{\tau^{*}}
$$



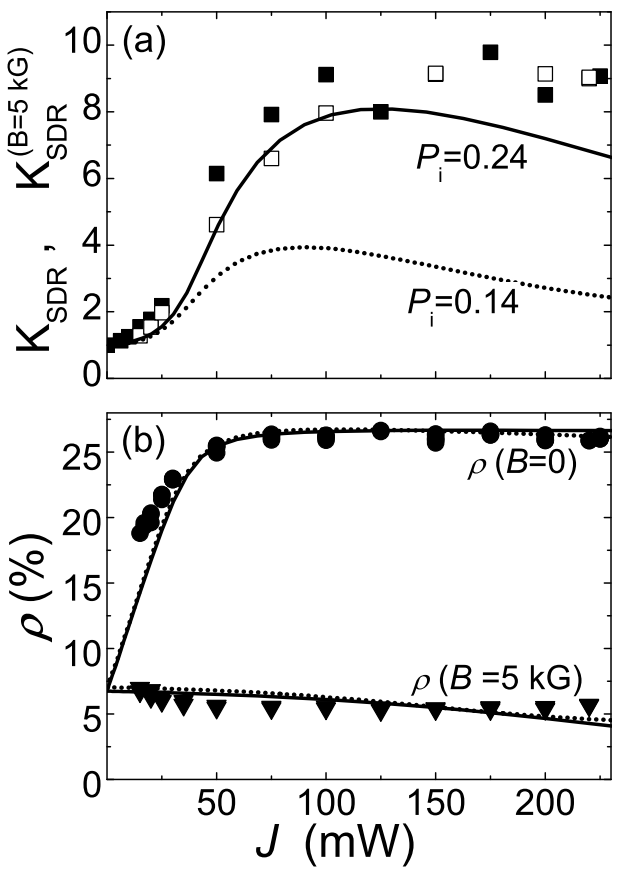

Figure 2. Spin-dependent recombination ratios (a) and the photoluminescence circular polarization (b) in $\mathrm{GaAs}_{0.979} \mathrm{~N}_{0.021}$ vs. the excitation power for the incidentphoton energy $\hbar \omega_{\text {exc }}=1.312 \mathrm{eV}$, the detection energy $\hbar \omega_{\text {det }}=1.17 \mathrm{eV}$ and room temperature. Full and open squares stand for the measured values of $K_{\mathrm{SDR}}=$ $I(\operatorname{circ}) / I(\operatorname{lin})$ and $K_{\mathrm{SDR}}^{(B=5 \mathrm{kG})}=I(\operatorname{circ}, B=0) / I(\operatorname{circ}, B=5 \mathrm{kG})$, respectively. Circles and triangles present values of the degree of circular polarization measured in the absence of magnetic field and at $B=5 \mathrm{kG}$. Solid and dotted lines are the result of the model calculation (see text for details).

\section{Comparison with experiment and discussion}

One of the most convincing signatures of spin-dependent recombination is dependence of the PL intensity on the light polarization at zero magnetic field, or more precisely, an increase in the PL intensity under the switching from linear to circular polarization of the exciting light. In figure 2 full squares show the measured SDR ratio defined by $K_{\mathrm{SDR}}=I(\operatorname{circ}, B=0) / I(\operatorname{lin}, B=0)$, where $I(\operatorname{circ}, B=0)$ and $I(\operatorname{lin}, B=0)$ are the PL intensity under circularly- and linearly-polarized photoexcitation. At very weak pumping power, the intensities $I$ (circ) and $I$ (lin) coincide. With increasing the power the PL intensity $I(\operatorname{circ}, B=0)$ is enhanced as compared with $I(\operatorname{lin}, B=0)$ and, at $J>100 \mathrm{~mW}$, the SDR ratio reaches values as high as 8 and even higher. A transverse magnetic field of $5 \mathrm{kG}$ strength eliminates the enhancement caused by the circular polarization of the incident light and, as a result, the modified SDR ratio $K_{\mathrm{SDR}}^{(B=5 \mathrm{kG})}=I(\operatorname{circ}, B=0) / I(\operatorname{circ}, B=5 \mathrm{kG})$ behaves as a function of $J$ identically to the dependence $K_{\mathrm{SDR}}(J)$. Simultaneously the degree of PL circular polarization induced under circularly-polarized photoexcitation (full circles in figure 2) monotonously increases up to $25 \%$ which is another important manifestation of the spin-dependent recombination. 

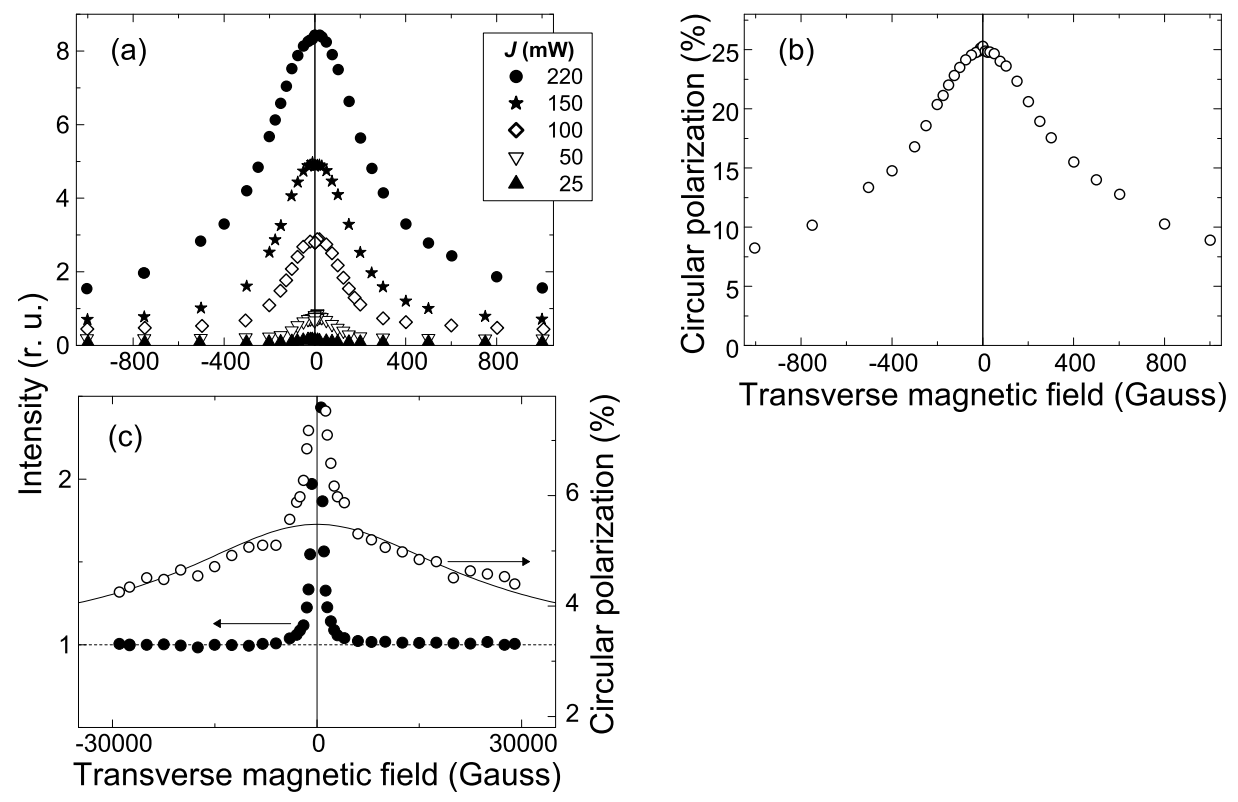

Figure 3. The photoluminescence intensity (a, c) and circular polarization (b, c) as functions of a transverse magnetic field measured in $\mathrm{GaAs}_{0.979} \mathrm{~N}_{0.021}$ for different powers of the circularly-polarized pumping. Experimental points presented in panel (a) are obtained for the following incident-light intensity $J$ (in $\mathrm{mW}$ ): $\mathbf{\Delta}-25, \nabla-$ $50, \diamond-100, \star-150, \bullet-220$. Panel (b) shows the upper part of the Hanle curve at $J=220 \mathrm{~mW}$ and panel (c) shows the dependencies $I(B)$ (solid circles) and $\rho(B)$ (open circles) measured at $J=220 \mathrm{~mW}$ in the wide range of magnetic fields. Solid line in panel (c) is the Lorentzian $\rho(B)=\rho_{0} /\left(1+B^{2} / B_{1 / 2}^{2}\right)+\rho_{\text {res }}$ with $B_{1 / 2}=25000 \mathrm{G}$. $\hbar \omega_{\mathrm{exc}}=1.312 \mathrm{eV}, \hbar \omega_{\mathrm{det}}=1.17 \mathrm{eV}$.

Figure 3 shows typical curves of magnetic-field-induced quenching of the photoluminescence (panels (a) and (c)) and its depolarization (panels (b) and (c)) in $\mathrm{GaAs}_{0.979} \mathrm{~N}_{0.021}$ measured under circularly-polarized pumping. One can see that the depolarization curve (Hanle effect) is composed of narrow and wide contours with the half-widths differing by two orders of magnitude. The main variation of narrow Hanle contour takes place at the same magnetic field as for the switching off the PL intensity enhancement which is a clear evidence that the narrow and wide parts of the Hanle curve are related to the spin depolarization of bound and free electrons, respectively.

For a quantitative description of the data presented in figures 2 and 3 we apply the two-charge-state model for the selected set of parameter values, i.e.,

$$
\begin{aligned}
& g=1, \quad g_{c}=2, \quad \tau^{*}=\left(c_{n} N_{c}\right)^{-1}=1.9 \mathrm{ps}, \\
& \tau_{h}^{*}=\left(c_{p} N_{c}\right)^{-1}=27 \mathrm{ps}, \quad \tau_{s}=140 \mathrm{ps}, \quad \tau_{s c}=700 \mathrm{ps} .
\end{aligned}
$$

Three remaining parameters of the theory, $P_{i}, P^{\prime}$ and $N_{c}$, are determined from comparison with the experimental data displayed in figures 2 and 3 . According to equations (30) and (32) values of $P_{i}$ and $P^{\prime}$ can be found as soon as the effective "residual" polarization $\rho_{\text {res }}$ is estimated. Unfortunately, the range of used magnetic field is insufficient to determine $\rho_{\text {res }}$ unambiguously: its value clearly lies in the 


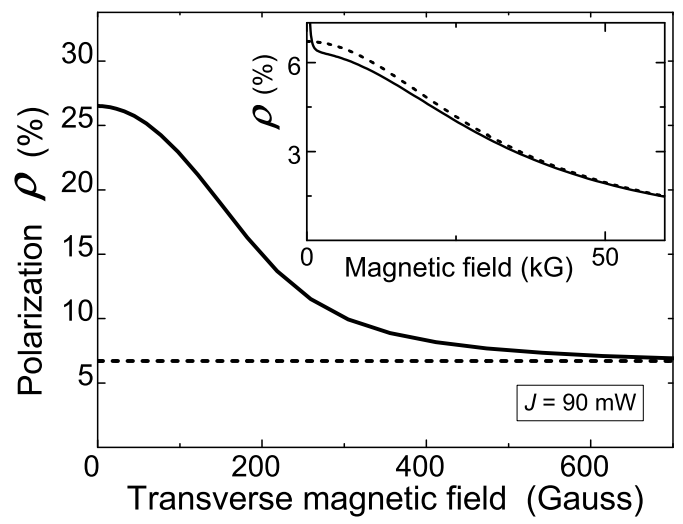

Figure 4. Calculated Hanle curve for the free-electron spin polarization $\rho$ in a coupled spin system of free and bound photoelectrons. Inset shows the larger magnetic-field range. Solid curve represents the exact calculation while dashed curve is obtained assuming unpolarized bound electrons in which case the spin filter is switched off.

interval between 0 and $3.3 \%$. Taking into account that, as follows from figure 2(b), $\rho_{\exp }(B=0$, high $J) \approx 25 \%$ and $\rho_{\exp }(B=5 \mathrm{kG}) \approx 6.7 \%$ and using equations (30), (32) we obtain $P^{\prime} \approx 0.25$ and $P_{i} \approx 0.27$ (if $\rho_{\text {res }}=0$ ) and $P^{\prime} \approx 0.22$ and $P_{i} \approx 0.16$ (if $\rho_{\text {res }}=3.3 \%$ ). An additional variation of these values follows from the difference of $P(B=0$, high $J)$ from unity since the ratio $\tau^{*} / \tau_{s}$ is small but finite. The selfconsistent calculation, see figure 6, shows that one should substitute into equation (32) the value $P(B=0$, high $J)=0.9$ so that the values $P^{\prime}=0.25, P_{i}=0.27$ are replaced by $P^{\prime}=0.28, P_{i}=0.24$. Due to the same reason, the values of $P^{\prime}, P_{i}$ corresponding to $\rho_{\text {res }}=3.3 \%$ should also be changed from $0.22,0.16$ to $0.27,0.14$. The curves in figure 2 are calculated for the two pairs of $P^{\prime}, P_{i}$ values with $N_{c}$ being the final fitting parameter. One can see that the set

$$
P_{i}=0.24, \quad P^{\prime}=0.28 \quad \text { and } \quad N_{c}=3 \times 10^{15} \mathrm{~cm}^{-3}
$$

rather well simulate the experimental dependencies.

The complete set (36), (37) of parameters allows us to simulate the effect of transverse magnetic field and analyze consequences of variation of each parameter. The theoretical Hanle curve calculated at pump power $J=90 \mathrm{~mW}$ is shown in figure 4 in the narrow range up to $700 \mathrm{G}$ and, in inset, in the wide range up to $60 \mathrm{kG}$. As mentioned in section 2, the Hanle curve outside the narrow contour is described by the Lorentzian (27). This property is demonstrated by drawing a subsidiary (dashed) line calculated assuming formally that paramagnetic centers are kept unpolarized, i.e., setting $\boldsymbol{S}_{c}=0$ in equations (4a) -(4e). Then the densities $n, N_{1}$ and $p$ are independent of the magnetic field and the polarization $\rho(B)$ is indeed given by the Lorentzian (27). In figure 4 the exact (solid) curve is rather well approximated by the dashed curve for $B>0.5 \mathrm{kG}$.

The two-charge-state model rather well reproduces manifestation of the Hanle effect in the PL intensity. The dependence of PL intensity on the transverse magnetic field calculated for different $\sigma$-pump intensities is shown in figure 5. In agreement with 


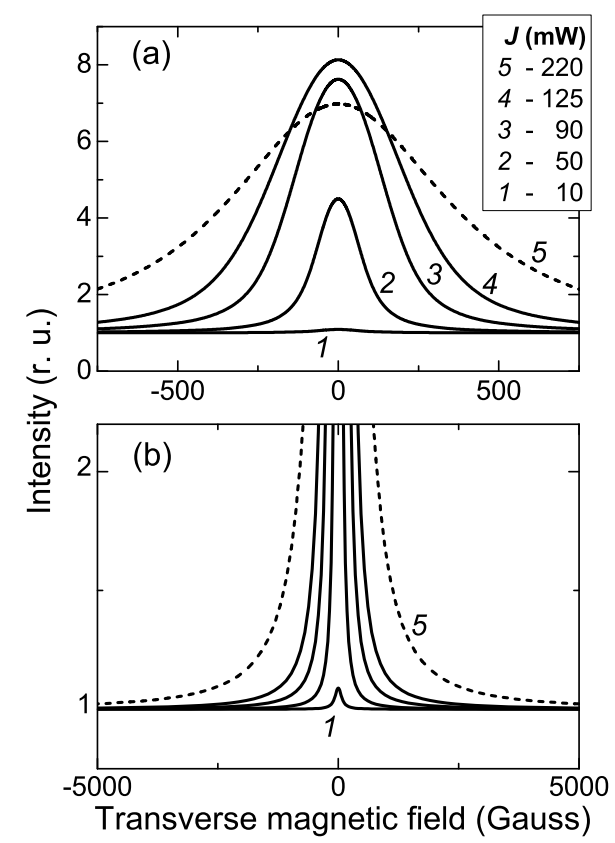

Figure 5. Magnetic-field dependence of the PL intensity $I(B)$ calculated at different powers $J$ of circularly polarized excitation and presented on small (a) and large (b) field scales. The curves $1 \ldots 5$ correspond to the following values of $J$ : $1-10 \mathrm{~mW}$, $2-50 \mathrm{~mW}, 3-90 \mathrm{~mW}, 4-170 \mathrm{~mW}, 5-220 \mathrm{~mW}$.

experiment the intensity $I$ decreases in relatively weak fields $|B|<5 \mathrm{kG}$ which suppress the polarization of bound electrons. Moreover, half-width of the decaying part of the function $I(B)$ is close to that of the narrow contour of the curve $\rho(B)$. At high fields $|B|>5 \mathrm{kG}$ gradually depolarizing the free electrons, the PL intensity is fieldindependent, see figure 5(b), in accordance with experimental data of figure 3(c).

Figure 6 displays the relation between the average electron spin polarization $P$ and the initial polarization $P_{i}$ for different pumping powers $J$. At low values of $J$ this relation is described by equation (23) which can be rewritten in the form

$$
P=\left(c_{0}+c_{1} \frac{J}{1 \mathrm{~mW}}\right) P_{i},
$$

where $c_{0}=T / \tau^{*}$ and $c_{1}=c_{0}^{2} \tau_{s c} G^{\text {opt }}(1 \mathrm{~mW}) / N_{c}$. Thus, at weak pumping and small $P_{i}$, the spin polarization $P$ is proportional to $P_{i}$ with the proportionality coefficient being a linear function of $J$. This approximate relation is shown in figure 6 by dotted straight lines. One can see that, at small $J$, the onset slopes of the exact and approximate curves $P\left(P_{i}\right)$ coincide; at moderate pumping the slopes slightly deviate, and only at large values of $J$ the difference between slopes gets remarkable. With increasing $P_{i}$ the dependence $P\left(P_{i}\right)$ at finite fixed $J$ becomes sublinear and finally saturates to $\tau_{s} /\left(\tau+\tau_{s}\right) \approx 1$.

Figure 7 illustrates effect of the sign of free-electron $g$ factor on the Hanle curve. The curves in the left and right panels are calculated for $g=1$ and $g=-1$, respectively, while the parameter $g_{c}=2$ is kept constant. One can see that, the value of $\rho(B=5 \mathrm{kG}$ ) (which determines the amplitude of the wide Hanle contour) calculated at $g=1$ remarkably 


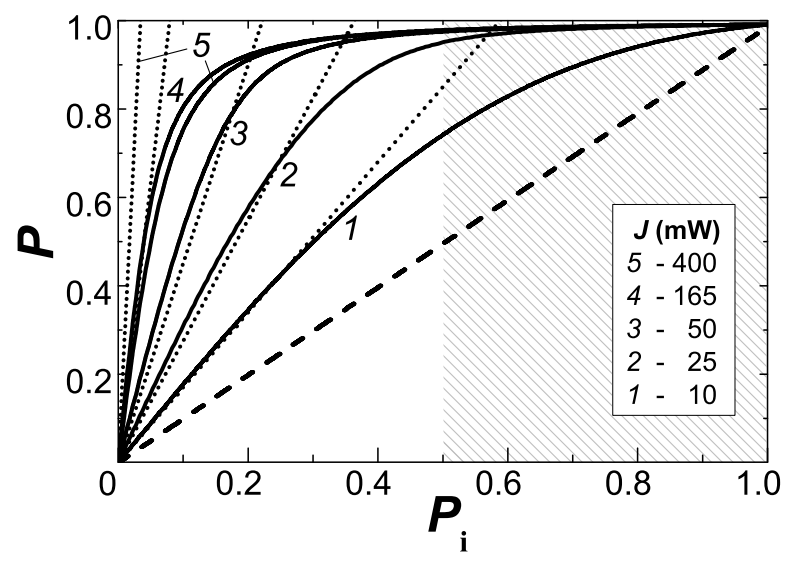

Figure 6. Relation between average spin polarization, $P$, of the conduction photoelectrons and their initial spin polarization $P_{i}$ calculated for different excitation powers varying from $10 \mathrm{~mW}$ to $400 \mathrm{~mW}$. The dashed line shows the limiting relation $P=P_{i} \tau_{s} /\left(\tau+\tau_{s}\right) \approx P_{i}$ attainable at vanishingly small intensity where the spin filter is switched off. The dotted straight lines represent the linear approximation (38). The shaded area $P_{i}>0.5$ is unachievable in a bulk undeformed semiconductor.

decreases with the increasing $J$. In contrast, at $g=-1$ the wide contour moves upwards as the intensity $J$ increases from $10 \mathrm{~mW}$ to $250 \mathrm{~mW}$ and, moreover, the narrow contour becomes much wider as compared to the case of $g=1$. This can be understood taking into account that, according to equation (91), in the system of coupled free and bound electron spins they precess in the transverse magnetic field faster if the signs of $g$ and $g_{c}$ coincide and slower if the signs of $g$ and $g_{c}$ are opposite. As a result, for negative $g$ and positive $g_{c}$ the function $\Psi$ in equation (8) becomes more robust to the influence of the transverse magnetic fields in the region $B \leq 2 \mathrm{kG}$. Comparison of the experimental Hanle curves in figure 2 of reference [36] with figures 7 (b) and (d) confirms the positive sign of $g$ determined in $\mathrm{GaAs}_{0.979} \mathrm{~N}_{0.021}$ under oblique pumping and detection [37.

The behaviour of the system under study in the wide range of pump intensities is demonstrated in figure 8. At weak intensities the dependence of $N_{1}$ and $N_{2}$ is insensitive to the polarization of incident light: $Y=N_{2} / N_{c}=\left(N_{c}-N_{1}\right) / N_{c} \propto \sqrt{J}$. With increasing $J$ the dependence $N_{2}(J)$ tends to saturation. For $\tau^{*}=1.9 \mathrm{ps}, \tau_{s c}$ $=700 \mathrm{ps}$ and $P_{i}=0.24$, we have for the critical values (34), (35) of the generation rates: $G_{\mathrm{cr}, \text { lin }}^{\mathrm{opt}} \gg G_{\mathrm{cr}, \text { circ }}^{\mathrm{opt}}$. Therefore, the deviation from the square-root law begins earlier for the circular polarization. The limiting values of $Y$ at $G^{\text {opt }} \rightarrow \infty$ are the solution $Y_{\infty}($ circ $)=0.72$ of equations (24) and the solution $Y_{\infty}(\operatorname{lin})=c_{n} /\left(c_{n}+c_{p}\right)=0.93$ of equation (28) at $X \rightarrow \infty$. The limiting values of $K_{\mathrm{SDR}}$ and $\rho$ are $\left[(1+a) Y_{\infty}(\mathrm{circ})\right]^{-2}=1.7$ and $\left(P^{\prime} P_{i} \tau_{s c} / \tau_{h}^{*}\right) Y_{\infty}(\mathrm{circ})=0.26$, respectively. The free-electron spin polarization tends to $P_{\infty}=\left(P_{i} \tau_{s c} / \tau_{h}^{*}\right) Y_{\infty}($ circ $)=0.9$. Note, however, that these limiting values are achieved at very high intensities far exceeding $J=1000 \mathrm{~mW}$ where the model defined by the parameter set (36), (37) is scarcely applicable. Particularly, an increase in the photoelectron and photohole densities at very high generation rates can lead to a remarkable contribution of the interband electron-hole recombination and the term 


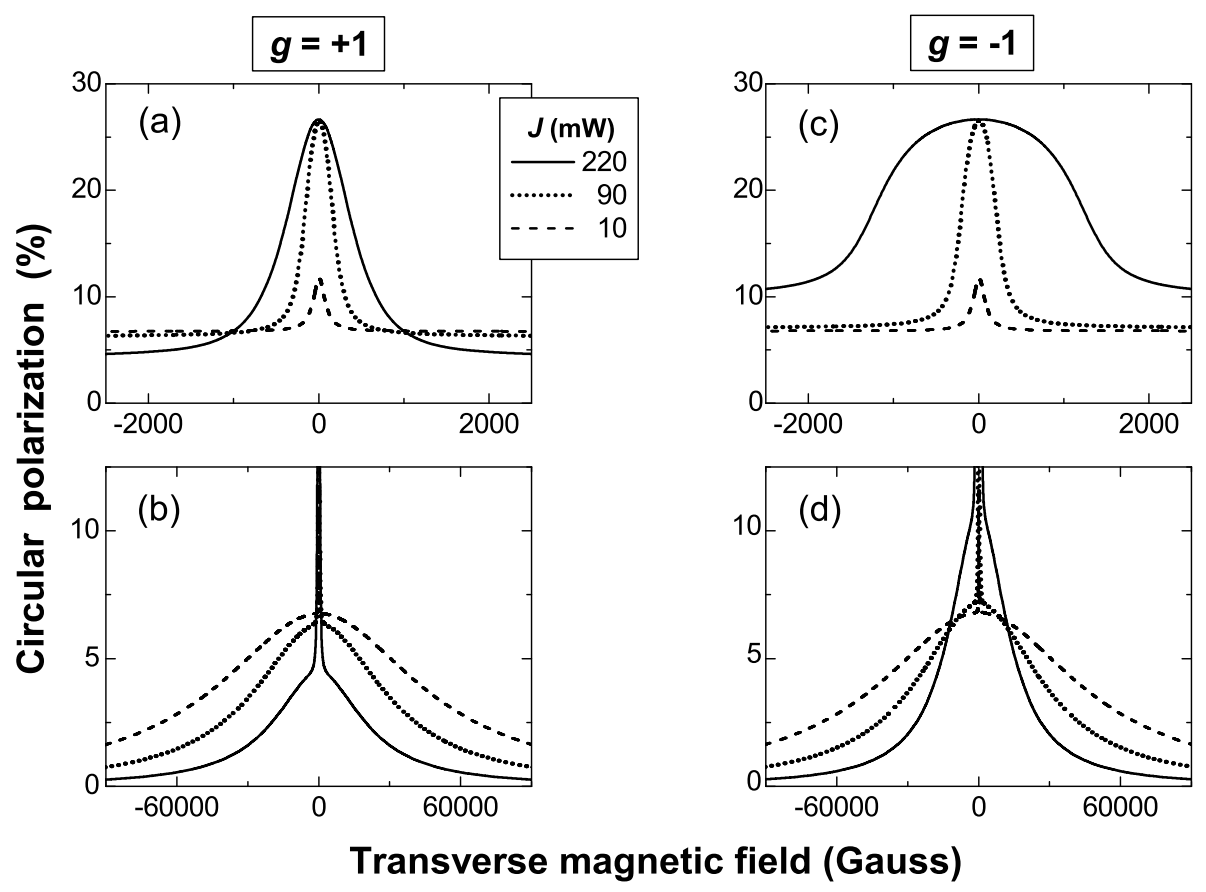

Figure 7. Hanle curves calculated for positive, $g=+1$ (a, b), and negative, $g=-1$ (c, d), values of the conduction electron $g$ factor and for different excitation powers $J=10 \mathrm{~mW}$ (dashed), $90 \mathrm{~mW}$ (dotted) and $250 \mathrm{~mW}$ (solid). Other parameters are indicated in text, see (36) and (37).

$\gamma n_{ \pm} p$ in equation (21) should be taken into consideration. A nonmonotonic behavior of $K_{\mathrm{SDR}}$ as a function of $J$ is an interesting and intuitively unexpected. In figure 2 experimental points only begin to show such behaviour. However, references [20, 23] report observation of a maximum in the dependence $K_{\mathrm{SDR}}(J)$. As one can see from figure 8(b), the degree of PL circular polarization rapidly increases with increasing $J$

and reaches a maximum value $\rho_{\max }=0.27$, then it almost stops to depend on $J$ slowly decreasing to $\rho_{\infty}=0.26$. The corresponding values of $P$ are $P_{\max }=0.94$ and $P_{\infty}=0.90$.

\section{Conclusion}

In summary, we have developed a spin-dependent theory of Shockley-Read recombination for three- and two-charge-state defects. The two-charge-state model characterized by nine parameters satisfactorily describes the coupled spin system of free and bound photoelectrons in undoped GaAsN alloys. In the weak- and strong-pumping limits, we present simple analytic equations which allow us to predict many properties of the coupled system not applying to computer simulations. Here we have concentrated on cw optical excitation. The further step could be a comprehensive analysis of timeresolved kinetics, including PL decay after short-pulse photoexcitation and pump-probe spectroscopy. It might be also interesting to study the strong-pumping regime where 

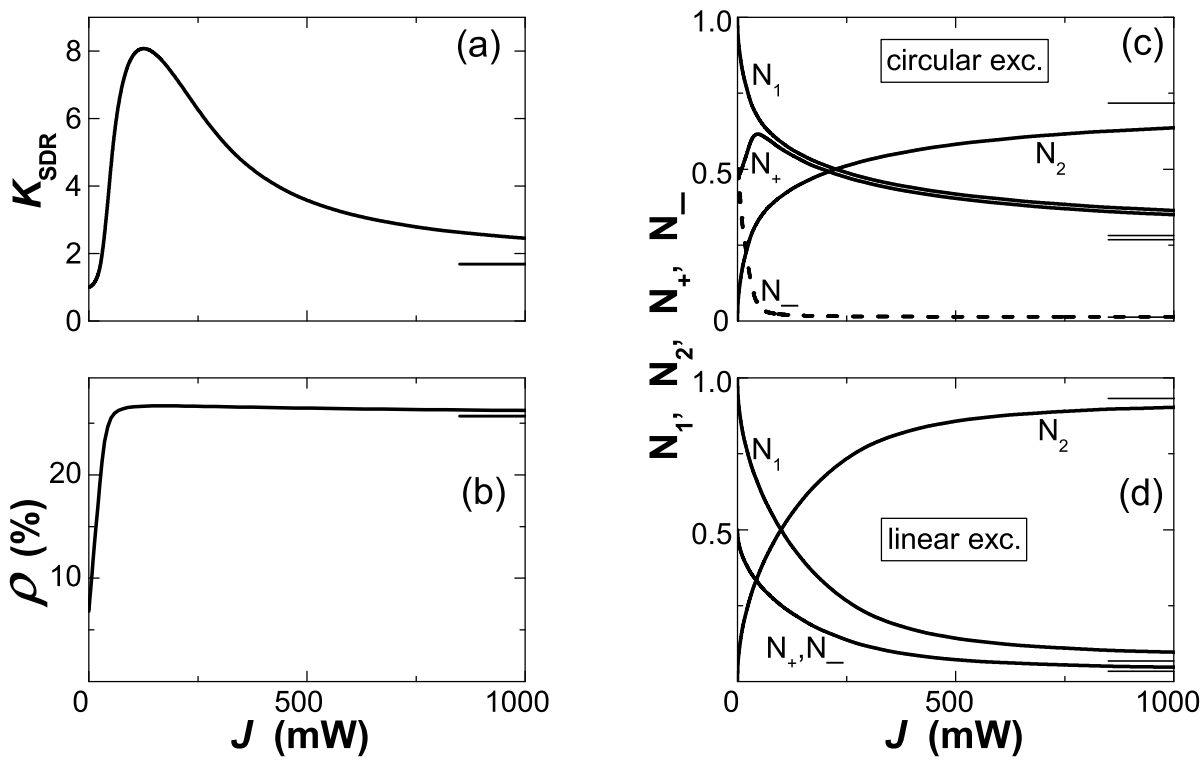

Figure 8. Variation of $K_{\mathrm{SDR}}, \rho, N_{1} / N_{c}, N_{2} / N_{c}, N_{+} / N_{c}$ and $N_{-} / N_{c}$ with the increasing pump intensity $J$ calculated in the wide range of $J$ for the set of parameters (36) and (37). Short horizontal lines indicate the limiting values at $J \rightarrow \infty$.

the recombination of free electrons and holes acquires an important role in the kinetics of photocarriers and work out predictions of specific behaviour of the system in this regime. Experimentally, it would be rather instructive to demonstrate unambiguously an existence or nonexistence of the deep-center level in GaAsN with no electron on the center.

\section{Acknowledgments}

This research was supported by the programmes of the Russian Academy of Sciences, Russian Foundation for Basic Research and TARA project of the University of Tsukuba. We are grateful to A Yu Egorov and V M Ustinov for useful discussions.

\section{References}

[1] Awschalom D D, N. Samarth N and Loss D (eds) 2002 Semiconductor Spintronics and Quantum Computation (Berlin: Springer-Verlag)

[2] Takagahara T (ed) 2003 Quantum coherence, correlation and decoherence in semiconductor nanostructures (London, San-Diego: Academic Press)

[3] Dyakonov M I (ed) 2008 Spin Physics in Semiconductors (Berlin: Springer)

[4] Kavokin K V 2008 Semicond. Sci. Technol. 23114009

[5] Meier F and Zakharchenya B P (eds) Optical orientation (Amsterdam: North-Holland)

[6] Song Pil Hun and Kim K W 2002 Phys. Rev. B 66035207

[7] Murdin B N et al 2005 Phys. Rev. B 72085346

[8] Glazov M M and Ivchenko E L 2001 JETP Lett. 74 380; 2004 JETP 991279

[9] Brand M, Malinowski A, Karimov A O, Mardsen P, Harley R, Shields A, Sanvitto D, Ritchie D and Simmons M 2002 Phys. Rev. Lett. 89236601 
[10] Wu M W, Jiang J H and Weng M Q 2010 Phys. Rep. 49361

[11] Kalevich V K, Ikezawa M, Shiryaev A Yu, Egorov A Yu, Ustinov V M and Masumoto Y 2003 Abstracts of the Physical Society of Japan (Autumn Meeting, Okayama, Japan, 20-23 September 2003) vol 58, iss 2, part 4, p 574

[12] Egorov A Yu, Kalevich V K, Afanasiev M M, Shiryaev A Yu, Ustinov V M, Ikezawa M and Masumoto Y 2005 J. Appl. Phys. 98013539

[13] Kalevich V K, Ivchenko E L, Afanasyev M M, Shiryaev A Yu, Egorov A Yu, Ustinov V M, Pal B and Masumoto Y 2005 JETP Lett. 82455

[14] Lombez L, Braun P-F, Carrère H, Urbaszek B, Renucci P, Amand T, Marie X, Harmand J C and Kalevich V K 2005 Appl. Phys. Lett. 87252115

[15] Kalevich V K, Ivchenko E L, Shiryaev A Yu, Egorov A Yu, Lombez L, Lagarde D, Marie X and Amand T 2007 AIP Conf. Proc. 8931309 (Proc. 28th Int. Conf. on Physics of Semiconductors, 2006, Vienna)

[16] Kalevich V K, Ivchenko E L, Shiryaev A Yu, Egorov A Yu, Lombez L, Lagarde D, Marie X and Amand T 2007 JETP Lett. 85174

[17] Lagarde D, Lombez L, Marie X, Balocchi A, Amand T, Kalevich V K, Shiryaev A, Ivchenko E and Egorov A 2007 Phys. Status Solidi a 204208

[18] Marie X, Lagarde D, Kalevich V K and Amand T 2008 Dilute III-V Nitride Semiconductors and Material Systems (Springer Series in Material Science vol 105) ed Erol A (Berlin-HeidelbergNew York: Springer) pp 283-299

[19] Puttisong Y, Wang X J, Buyanova I A, Carrère H, Zhao F, Balocchi A, Marie X, Tu C W and Chen W M 2010 Appl. Phys. Lett. 96, 052104

[20] Zhao F, Balocchi A, Truong G, Amand T, Marie X, Wang X J, Buyanova I A, Chen W M and Harmand J C 2009 J. Phys.: Condens. Matter 21174211

[21] Wang X J, Puttisong Y, Tu C W, Ptak Aaron J, Kalevich V K, Egorov A Yu, Geelhaar L, Riechert H, Chen W M and Buyanova I A 2009 Appl. Phys. Lett. 95241904

[22] Zhao F, Balocchi A, Kunold A, Carrey J, Carrère H, Amand T, Abdallah N Ben, Harmand J C and Marie X 2009 Appl. Phys. Lett. 95241104

[23] Zhao F, Balocchi A, Kunold A, Carrey J, Carrère H, Amand T, Abdallah N Ben, Harmand J C and Marie X 2009 arXiv:090\%.4321v1

[24] Wang X J, Buyanova I A, Zhao F, Lagarde D, Balocchi A, Marie X, Tu C W, Harmand J C and Chen W C 2009 Nature Materials 8198

[25] Weisbuch C and Lampel G 1974 Solid State Commun. 14141

[26] Paget D 1984 Phys. Rev. B 30931

[27] Holland M G and Paul W 1962 Phys. Rev. 12843

[28] Harris R D, Newton J L and Watkins G D 1987 Phys. Rev. B 361094

[29] Buse K and Krátzig E 1995 Appl. Phys. 6127

[30] Huot N, Jonathan J M C and Roosen G 1997 Appl. Phys. 65489

[31] Malouin M-A, El-Mellouhi F and Mousseau N 2007 Phys. Rev. B 76045211

[32] Shockley W and Last J T 1957 Phys. Rev. 107392

[33] Shockley W and Read W T 1952 Phys. Rev. 87835

[34] Abakumov V N, Perel V I and Yassievich I N 1991 Nonradiative Recombination in Semiconductors (Amsterdam: North-Holland)

[35] Asnin V M, Bir G L, Lomasov Yu N, Pikus G E and Rogachev A A 1976 Sov. Phys. JETP 44838

[36] Kalevich V K, Shiryaev A Yu, Ivchenko E L, Afanasiev M M, Egorov A Yu, Ustinov V M and Masumoto Y 2009 Physica B 4044929

[37] Kalevich V K, Ivchenko E L, Shiryaev A Yu, Afanasiev M M, Egorov A Yu, Ikezawa M and Masumoto Y 2008 Semicond. Sci. Technol. 23114008

[38] Pettinari G et al 2006 Phys. Rev. B 74245202

[39] O’Reilly E P, Lindsay A, Klar P J, Polimeni A and Capizzi M 2009 Semicond. Sci. Technol. 24 033001 
[40] Shan W, Walukiewicz W, Ager III J W, Haller E E, Geisz J F, Friedman D J, Olson J M and Kurtz S R 1999 Phys. Rev. Lett. 821221

[41] Skierbiszewski C, Pfeffer P and Łusakowski J 2005 Phys. Rev. B 71205203

[42] Masia F et al 2006 Phys. Rev. B 73073201

[43] Yang C L, Dai Junfeng, Ge W K and Cui Xiaodong 2010 Appl. Phys. Lett. 96152109

[44] Zhao H M, Lombez L, Liu B L, Sun B Q, Xue Q K, Chen D M and Marie X 2009 Appl. Phys. Lett. 95041911

[45] Bi W G and Tu C W 1977 Appl. Phys. Lett. 701608

[46] Tisch U, Finkman E and Salzman J 2002 Appl. Phys. Lett. 81463 